\title{
Quantification of growth factors by using a new system for obtaining platelet-rich plasma
}

\author{
Antonio Lorente-Pérez-Sierra ${ }^{1}$, Ricardo Ortega-Aranegui ${ }^{2}$, María Martín-Ares ${ }^{3}$, Juan López-Quiles-Mar- \\ tínez ${ }^{4}$, José-Ma Martínez-González ${ }^{5}$
}

\footnotetext{
${ }^{1}$ Associate Professor. School of Dentistry. European University of Madrid

${ }^{2}$ Associate Professor. School of Dentistry. Complutense University of Madrid

${ }^{3}$ Student in the Implant and Oral Surgery master's degree program. School of Dentistry. Complutense University of Madrid

${ }^{4}$ Senior Lecturer. School of Dentistry. Complutense University of Madrid Hospital La Zarzuela

${ }^{5}$ Full Professor. School of Dentistry. Complutense University of Madrid. Head of the Department of Oral Surgery and Implantology. University Hospital of Madrid
}

Correspondence:

School of Dentistry,

Complutense University of Madrid,

Pza. Ramón y Cajal s/n,

28040 Madrid

jmargo@odon.ucm.es

Received: 01/05/2010

Accepted: 30/09/2010
Lorente-Pérez-Sierra A, Ortega-Aranegui R, Martín-Ares M, LópezQuiles-Martínez J, Martínez-González JM. Quantification of growth factors by using a new system for obtaining platelet-rich plasma Med Oral Patol Oral Cir Bucal. 2011 Jul 1;16 (4):e614-8.

http://www.medicinaoral.com/medoralfree01/v16i4/medoralv16i4p614.pdf

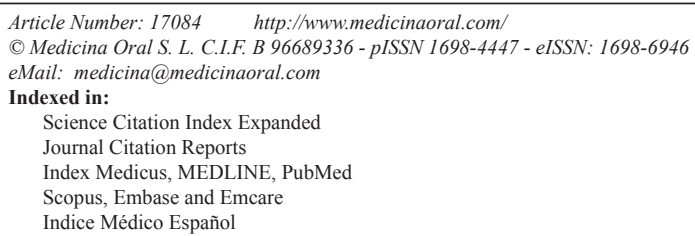

\begin{abstract}
Objective: To verify the performance of a new method for obtaining platelet-rich plasma, while avoiding contamination of the sample during its processing.

Study Design: Twenty healthy patients were selected, from whom $21 \mathrm{ml}$ of blood was extracted. We then proceeded to study the platelets and growth factors in basal blood after centrifuging the sample by using a new closed system for obtaining platelet-rich plasma (PRP).

Results: After centrifuging the blood sample, double the amount of platelets as that found in basal blood was obtained. Of the four growth factors analyzed, only the factor similar to insulin (IGF) contained the same concentration after the centrifuge process. The platelet-derived growth factor (PDGF) and the vascular growth factor (VGF) were multiplied by six with respect to the basal values and disproportionately increased the levels of the transforming growth factor $\beta$ (TGF- $\beta$ ).

Conclusions: The new closed method for obtaining PRP, after avoiding contamination of the sample following its use, offers levels of platelet concentrate and growth factors necessary for regeneration.
\end{abstract}

Key words: Platelet-rich plasma, growth factors, centrifuge. 


\section{Introduction}

Platelet activation induces degranulation of the alpha granules that contain growth factors. Of all the growth factors, those that are most involved in healing are the transforming growth factor $\beta$ (TGF- $\beta$ ), the factor similar to insulin (IGF), the platelet-derived growth factor (PDGF) and the vascular growth factor (VGF).

Platelet-rich plasma enables obtaining a high concentration of growth factors. Its clinical application triggers acceleration in the healing of soft tissues and bone neoformation (1). Marx (2) studied the effect of platelet concentrate on the cells of the periodontal ligament in vitro, observing an increase in cell division and the secretory function. Karl et al. (3) applied concentrates of PRP to bone cell cultures, obtaining an increase in the percentage of proliferation and cell migration.

In the scientific literature, there is a large debate about the systems for obtaining PRP, in terms of the platelet concentrate and the integrity of the platelets during the handling and processing $(4,5)$.

The objective of this study was the analysis of the effectiveness of a new closed method for obtaining platelet concentrate and growth factors, in which exposure of the blood to the external environment is avoided and the platelets are maintained in tact until the moment they are used.

\section{Material and Method}

Obtaining and processing of the sample

Twenty healthy volunteer patients were selected, with an age ranging from 19 to 23 years old. A peripheral extraction of blood was performed (cephalic vein). For the puncture, the Multyfly $21 \mathrm{G}$ system was used, which was connected to a $3 \mathrm{mI} 9 \mathrm{NC}$ Monovette syringe $(3.30 \mathrm{ml}$ of Stardet ${ }^{\circledR}$ sodium citrate). Aspiration was performed gradually, given that it is less traumatic and fewer cells are destroyed. After the extractions, the plastic stem on the syringe was broken and introduced with the cap pointing downwards in the inserts of the centrifuge.

The sample was processed in a Bayer ${ }^{\circledR}$ conventional laboratory centrifuge, making a first centrifuge with $800 \mathrm{~g}$ for a period of 2 minutes, in order to eliminate the heavier elements (red blood cells). Once this was accomplished, the samples were placed face down in the rack, finding two clearly distinguished phases, a lower part of the tube with red blood cells and another, on top, with the plasma. The red phase was eliminated by connecting a Multyfly ${ }^{\circledR}$ device and the plasma was centrifuged at $500 \mathrm{~g}$ for 8 minutes. Three distinct phases were obtained from this second centrifugation. The first contained residual red blood cells and leukocytes; the second phase consisted of plasma rich in concentration of platelets, and the third phase consisted of platelet-poor plasma. The first phase was aspirated again in order to dispose of it, and the rest of the tube was decanted in order to be analyzed.

\section{Count and determination}

For each patient, we performed a count of basal platelets in the entire blood using the analyzer Cell-Dyn 3500 and determined the growth factors (PDGF, TGF- $\beta$, VGF) by means of ELISA from the laboratory Bender MedSystem $^{\circledR}$ and the IGF was determined by an enzymatically amplified chemiluminescent immunometric assay of the laboratory Diagnostic Products Corporation ${ }^{\circledR}$. After the centrifuge, we analyzed the part rich in platelets, with the platelet count and determination of the same growth factors as that analyzed in all of the blood.

We extracted $21 \mathrm{ml}$ of blood from each volunteer, which was placed into 7 tubes, each one with $3 \mathrm{ml}$. Upon centrifuge, we obtained a resultant tube which contained between 4 to $5 \mathrm{ml}$ of PRP. The sample was doubly diluted, pouring out more blood serum in order to achieve a minimum volume in order to make counts without extracting a large volume of blood from the patient.

The Student T test was used as a parametric test for the statistical analysis, obtaining statistic significance for $\mathrm{p}<0.05$ and the test of the ranges with the Wilcoxon sign as non-parametric, in order to study the statistic significance of the results.

\section{Results}

By applying the Student-T test, we are able to affirm that the changes in concentration were significant $p<0.001$ in all of the parameters studied except in the TGF- $\beta$, in which it is not clearly significant due to the high typical deviation. After contrasting the ranges with the Wilcoxon sign for matching data, we learned that from the 20 samples in which the concentration of platelets was measured, it increased in 18 samples and decreased in only 2 samples $(p<0.001)$. With respect to the concentration of the growth factors, there was an increase in IGF in only 8 samples, whereas the concentration decreased in the other 12 samples $(p<0.05)$. The concentration of TGF- $\beta$ increased in 13 samples, remained the same in one of them and decreased in the rest $(p<0.001)$. In the 20 samples, there was an increase in PDGF $(p<0.001)$ and in VGF $(\mathrm{p}<0.001)$.

\section{Platelet count}

The values obtained for basal platelets were within the normal values, with a minimum of 94,000 and a maximum of $293,000 / \mathrm{mm}^{3}$. The average platelet count was $185,000 / \mathrm{mm}^{3}$, with a standard deviation of 43 , which indicates a very homogeneous sample. After centrifuging the samples, we observed an increase of approximately double in the count of concentrated platelets, given that the average was 354.71 , with a standard deviation of 128.8 .

\section{Count of growth factors}

For the same samples, the average concentration in basal blood of IGF was analyzed, which was 173.48 , with a standard deviation of 48.57 , of TGF- $\beta$ equal to 22.33 and 
Table 1. Values, in each one of the samples, of platelets per cubic millimeter of blood multiplied by 1000 (PLT-1; in basal blood and PLT-2; in concentrate). Count of the basal growth factors (IGF-1, TGFß-1, PDGF-1, VGF-1). Count of the concentrated growth factors (IGF-2, TGF $3-2$, PDGF-2, VGF-2) in each one of the 20 samples.

\begin{tabular}{|c|c|c|c|c|c|}
\hline PATIENT & $\begin{array}{c}\text { PLT-1 } \\
\text { /PLT-2 } \\
\left(10 X 3 / M^{3}\right)\end{array}$ & $\begin{array}{l}\text { IGF-1 } \\
\text { /IGF-2 } \\
\text { (ng/ml) }\end{array}$ & $\begin{array}{c}\text { TGF } \beta-1 \\
\text { /TGF } \beta-2 \\
\text { (ng/ml) }\end{array}$ & $\begin{array}{c}\text { PDGF-1 } \\
\text { /PDGF-2 } \\
\text { (pg/ml) }\end{array}$ & $\begin{array}{c}\text { VGF-1 } \\
\text { /VGF-2 } \\
\text { (pg/ml) }\end{array}$ \\
\hline 1 & $179 / 266$ & $252 / 256$ & $29.7 / 310.7$ & 486 / 3204 & $22.4 / 227.6$ \\
\hline 2 & $152 / 332$ & $126 / 122$ & $42.7 / 3.8$ & 454 / 3435 & $80.1 / 176.3$ \\
\hline 3 & $204 / 384$ & $179 / 162$ & $12 / 360$ & $453 / 2646$ & $147.4 / 320.3$ \\
\hline 4 & $293 / 451$ & $119 / 122$ & $13,6 / 0,1$ & $746 / 3657$ & $\begin{array}{c}1409.1 / \\
1592.2\end{array}$ \\
\hline 5 & $173 / 127$ & $127 / 142$ & $43.5 / 0.1$ & 913 / 3452 & $102.6 / 237.2$ \\
\hline 6 & $182 / 271$ & $214 / 220$ & $0.1 / 0.1$ & 344 / 3419 & $80.1 / 250$ \\
\hline 7 & $143 / 431$ & $171 / 132$ & $36.8 / 0.1$ & $374 / 3378$ & $67.3 / 359.4$ \\
\hline 8 & $132 / 280$ & $189 / 174$ & $0.1 / 32.7$ & 307 / 3035 & $3.2 / 121.8$ \\
\hline 9 & $157 / 296$ & $283 / 275$ & $1.6 / 296.5$ & $311 / 3110$ & $3.2 / 35.3$ \\
\hline 10 & $212 / 343$ & $237 / 233$ & $59.9 / 341$ & $1461 / 3613$ & $86.5 / 214.7$ \\
\hline 11 & $163 / 147$ & $132 / 131$ & $0.5 / 53.2$ & 1109 / 2466 & $16 / 86.5$ \\
\hline 12 & $139 / 506$ & 174 / 181 & $0.5 / 63.6$ & $366 / 3076$ & $28.8 / 691.4$ \\
\hline 13 & $142 / 282$ & $117 / 102$ & $42.7 / 309.6$ & 709 / 3400 & $25.6 / 484.4$ \\
\hline 14 & $205 / 612$ & 117 / 127 & $12.5 / 308.8$ & 219 / 3347 & $35.3 / 335.9$ \\
\hline 15 & $135 / 342$ & $133 / 124$ & $27.6 / 314.1$ & 442 / 3277 & $32.1 / 402.3$ \\
\hline 16 & $179 / 233$ & $96,4 / 120$ & $18 / 231.5$ & $155 / 3270$ & $125 / 429.7$ \\
\hline 17 & $221 / 490$ & $180 / 145$ & $0.1 / 360$ & 548 / 3439 & $76.9 / 507.8$ \\
\hline 18 & $218 / 282$ & $183 / 176$ & $22.2 / 10.4$ & 334 / 3165 & $64.1 / 359.4$ \\
\hline 19 & $232 / 534$ & $111 / 101$ & $10.4 / 79.5$ & 805 / 4000 & $70.5 / 410.2$ \\
\hline 20 & $214 / 683$ & $223 / 265$ & $37.7 / 1102$ & 494 / 4000 & 99.4 / 843.8 \\
\hline
\end{tabular}


standard deviation of 29.23. The PDGF was de 698.20, with a standard deviation of 487.26 , and the average concentration for VGF was 164.12, with a standard deviation of 287.04 .

Of the four growth factors analyzed, all of them were concentrated except the IGF, which was to be expected given that the platelets are not their storage place. Thus, the values obtained are equal or less than the basal values, with an average value of 166.73 and a standard deviation of 49.97 .

In the case of PDGF and VGF, the average concentrated values were six times higher than the basal values. The average of the PDGF reached 3181.52, with a standard deviation of 565.17. For VGF, the average was 423.38 and a standard deviation of 333.16. And in determining the TGF- $\beta$, the average was 512 times higher than in the basal states, due to the fact that there was a large range in the extreme values, from 0.10 to $1102 \mathrm{ng} / \mathrm{ml}$, with a standard deviation of 201.3 (Table 1).

\section{Discussion}

One of the most debated applications for platelet-rich plasma has been bone regeneration. In this regard, Cheng et al. (6), as other authors have discussed, arrive at the conclusion that PRP combined with stem cells form a composite that is capable of improving bone regeneration in critical defects generated in rabbits. With these same criteria, Nair et al. (7) arrive at the same conclusion with the exception of including a biomaterial such as hydroxyapatite in the mixture. Pieri et al. (8) obtain a higher percentage of bone formation with a mixture of stem cells and PRP in alveolar defects produced in the mandibles of mini-pigs.

In 2009, Plachokova et al. (9) carried out a systematic review in order to see the effect of PRP on bone regeneration in dentistry and arrive at the conclusion that it is useful in sinus lifts and in periodontal defects, contradicting the conclusions that it had obtained in 2006. In another study by Plachokova et al. (10) in 2009, they concluded that PRP does not present significant advantages in laboratory animals such as rats. However, human PRP in combination with biomaterials aid the healing of bone grafts.

There are also other studies that shed light on the possible secondary effects that may appear due to the indiscriminate use of plasma with platelets, offering a perspective that differs from the majority of articles published on the topic $(11,12)$.

In our study, as already mentioned, the concentrates were diluted in order to have a sufficient sample for determining the proteins, which is why our results and determinations, which are by unit of volume, are half of what would have been obtained if the correct amount of blood serum had been taken in order to be used in regeneration. The amount of blood serum for having a sufficient volume of concentrate would be $10 \mathrm{ml}$, given that the analyzer Cell Dyn needs a minimum volume of sample in order to determine the number of platelets, as well as for determining the growth factors. This was the justification for diluting the sample.

The average of the concentration of platelets obtained was double the count in peripheral blood, which resulted in a poorer result than that obtained with other methods that were studied (13). This analysis was carried out with the absolute values in the samples taken, which, as aforementioned, were diluted. This data may appear to be discouraging with respect to the other methods, given that many of them quadrupled the number of platelets. The concentration of IGF is practically the same in the concentrates as in the basal samples. The explanation for this result is probably that the platelets are not a deposit of IGF, and no matter how much they are concentrated, this procedure will not increase their amount. The average of the results in the concentration of TGF- $\beta$ is 521 times higher than that obtained by other authors when measuring the effectiveness of other methods (14). One of the reasons that such high values are obtained may be due to the extreme values that came out in the samples and that deviated the average $(11,000 \mathrm{ng} / \mathrm{ml})$, but even when those values are eliminated, it continues to be eight times higher than the basal value. This can be explained by the early state of activation of the platelets, which has prevented the deposits of such proteins from being released. Our results are similar, and in any case, better than those obtained in other studies. We may consider that, although the platelet concentration obtained is not the same as with other systems, a greater integrity of the platelets is maintained in our study, which enables the TGF deposits that are in the alpha granules to be kept intact until the end, without losing their content, being able to be released when they are used. The PDGF and the VGF were concentrated six times more than in the peripheral blood, which improves the results or at least matches those presented by other authors such as $\mathrm{Hu} \mathrm{Z}$ et al. (15).

The study conducted by Anitua (16) analyzes a sample that is almost identical to ours. It relates the concentrate of platelets with obtaining growth factors, concluding that there is variability in the concentration of VGF in different individuals, as also confirmed by our results. They are able to focus on the average, but they also report very different values, both excessive as well as a lack of concentration of platelets. The IGF concentration is totally independent from the number of platelets, given that their deposit in the platelets is minimal. The concentration of the other two growth factors ends up being between 2.5 and 3 times, whereas with our system, it varies between 6 times for PDGF and 8 for TGF, which improves the aforementioned results.

The four protein counts obtained using our method have 
achieved the results of other systems, despite the concentration of platelets being less in the majority of the systems, given that it is double and not quadruple. This caused us to consider that what was important was not the number of platelets, but the condition of these platelets and their function in order to be used if needed. It is logical that if the processing of the sample damages or activates the platelets, the content of its granules would be released prematurely, and as a consequence, would be lost before being used.

The study by Mazzucco et al. (17) in 2009 further supports this hypothesis. It analyzes four different methods for obtaining platelet-rich plasma. As has been demonstrated, the different methods have distinct centrifugation systems in terms of centrifugal strength, which makes us think that it is not a determining factor for concentrating the platelets. They also use different anticoagulants and gelification methods. Despite the different methodologies, all of the systems achieve a good concentration of platelets, but it is demonstrated that there is no direct relationship between the amount of platelets and the growth factor concentration. The conclusion of the author is that the handling of the samples causes damage to the platelets, such that they activate and release their content. This causes the clinical result to depend on the system used for obtaining the PRP.

The values that we obtained lead us to think that had the sample not been diluted, the results would have been better. In any case, the concentration of growth factors obtained is comparable to any other system, which would make it apt for being used in regeneration.

\section{References}

References with links to Crossref - DOI

1. Anitua E, Sánchez M, Orive G, Andía I. The potential impact of the preparation rich in growth factors (PRGF) in different medical fields. Biomaterials. 2007;28:4551-60.

2. Marx RE. Platelet-rich plasma: evidence to support its use. J Oral Maxillofac Surg. 2004;62:489-96.

3. Kark LR, Karp JM, Davies JE. Platelet releasate increases the proliferation and migration of bone marrow-derived cells cultured under osteogenic conditions. Clin Oral Implants Res. 2006;17:321-7.

4. Gonshor A. Technique for producing platelet-rich plasma and platelet concentrate: background and process. Int J Periodontics Restorative Dent. 2002;22:547-57.

5. Appel TR, Pötzsch B, Müller J, von Lindern JJ, Bergé SJ, Reich $\mathrm{RH}$. Comparison of three different preparations of platelet concentrates for growth factor enrichment. Clin Oral Implants Res. 2002;13:522-8.

6. Cheng X, Lei D, Mao T, Yang S, Chen F, Wu W. Repair of critical bone defects with injectable platelet rich plasma/bone marrowderived stromal cells composite: experimental study in rabbits. Ulus Travma Acil Cerrahi Derg. 2008;14:87-95.

7. Nair MB, Varma HK, Menon KV, Shenoy SJ, John A. Reconstruction of goat femur segmental defects using triphasic ceramic-coated hydroxyapatite in combination with autologous cells and plateletrich plasma. Acta Biomater. 2009;5:1742-55.

8. Pieri F, Lucarelli E, Corinaldesi G, Fini M, Aldini NN, Giardino $\mathrm{R}$, et al. Effect of mesenchymal stem cells and platelet-rich plasma on the healing of standardized bone defects in the alveolar ridge: a comparative histomorphometric study in minipigs. J Oral Maxillofac Surg. 2009;67:265-72.
9. Plachokova AS, Nikolidakis D, Mulder J, Jansen JA, Creugers NH. Effect of platelet-rich plasma on bone regeneration in dentistry: a systematic review. Clin Oral Implants Res. 2008;19:539-45.

10. Plachokova AS, Van den Dolder J, Van den Beucken JJ, Jansen JA. Bone regenerative properties of rat, goat and human platelet-rich plasma. Int J Oral Maxillofac Surg. 2009;38:861-9.

11. Martinez-Gonzalez JM, Cano-Sanchez J, Gonzalo-Lafuente JC, Campo-Trapero J, Esparza-Gomez G, Seoane J. Do ambulatory-use Platelet-Rich Plasma (PRP) concentrates present risks? Med Oral. 2002;7:375-90

12. Landesberg R, Moses M, Karpatkin M. Risks of using platelet rich plasma gel. J Oral Maxillofac Surg. 1998;56:1116-7.

13. Marx RE, Carlson ER, Eichstaedt RM, Schimmele SR, Strauss JE, Georgeff KR. Platelet-rich plasma: Growth factor enhancement for bone grafts. Oral Surg Oral Med Oral Pathol Oral Radiol Endod. 1998;85:638-46.

14. Appel TR, Pötzsch B, Müller J, Von Lindern JJ, Bergé SJ, Reich RH. Comparison of three different preparations of platelet concentrates for growth factor enrichment. Clin Oral Implants Res. 2002;13:522-8.

15. Hu Z, Peel SA, Ho SK, Sándor GK, Clokie CM. Platelet-rich plasma induces mRNA expression of VEGF and PDGF in rat bone marrow stromal cell differentiation. Oral Surg Oral Med Oral Pathol Oral Radiol Endod. 2009;107:43-8.

16. Anitua E. The use of plasma-rich growth factors (PRGF) in oral surgery. Pract Proced Aesthet Dent. 2001;13:487-93.

17. Mazzucco L, Balbo V, Cattana E, Guaschino R, Borzini P. Not every PRP-gel is born equal. Evaluation of growth factor availability for tissues through four PRP-gel preparations: Fibrinet, RegenPRPKit, Plateltex and one manual procedure. Vox Sang. 2009;97:110-8. 